\title{
Frontières
}

\section{Baud, René-Claude, Ce qui remonte de l'ombre. Itinéraire d'un soignant, Paris, Bayard, 2006, 163 p.}

\section{Jean-Jacques Lavoie}

Volume 19, numéro 2, printemps 2007

\section{Penser sa mort?}

URI : https://id.erudit.org/iderudit/017509ar

DOI : https://doi.org/10.7202/017509ar

Aller au sommaire du numéro

\section{Éditeur(s)}

Université du Québec à Montréal

ISSN

1180-3479 (imprimé)

1916-0976 (numérique)

Découvrir la revue

Citer ce compte rendu

Lavoie, J.-J. (2007). Compte rendu de [Baud, René-Claude, Ce qui remonte de l'ombre. Itinéraire d'un soignant, Paris, Bayard, 2006, 163 p.] Frontières, 19(2), 79-80. https://doi.org/10.7202/017509ar

Ce document est protégé par la loi sur le droit d'auteur. L'utilisation des services d'Érudit (y compris la reproduction) est assujettie à sa politique d'utilisation que vous pouvez consulter en ligne.

https://apropos.erudit.org/fr/usagers/politique-dutilisation/
Cet article est diffusé et préservé par Érudit.

Érudit est un consortium interuniversitaire sans but lucratif composé de l’Université de Montréal, l'Université Laval et l'Université du Québec à Montréal. Il a pour mission la promotion et la valorisation de la recherche. https://www.erudit.org/fr/ 
sommes poursuivis toute une vie par ce que nous n'avons pas osé vivre en entièreté » (p. 56); "Au cœur d'une maladie mortelle, on peut tout entreprendre avec le temps imparti » (p. 101); "Chaque jour se doit d'être une création nouvelle» (p. 114)

Tout en étant d'une lucidité, d'une intensité de vivre qui font mal, ce livre nous aide à vivre. Des romans et essais d'elle, il faut lire au moins, si ce n'est déjà fait, ses incontournables: La mort viennoise, Une passion, Les sept nuits de la reine, Seul ce qui brûle. C. Singer est décédée à Vienne, le 4 avril 2007. Mais elle vivra tant que nous la lirons. Lisons-la, encore et encore, et offrons ses livres aux jeunes qui se croient immortels.

Hans-Jürgen Greif

\section{HENTSCH, Thierry}

\section{Raconter et mourir}

\section{Aux sources narratives de l'imaginaire occidental}

Montréal, Les Presses de I'Université de Montréal, 2002, $431 \mathrm{p}$.

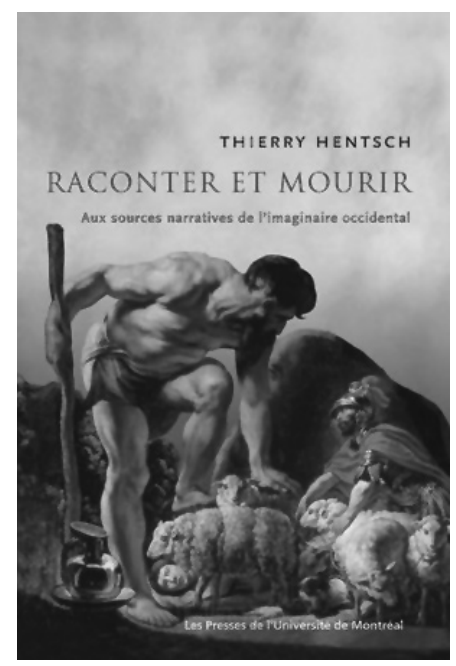

Dans l'introduction, l'auteur annonce ses couleurs en adoptant une posture qui démontre à l'envi tout au long du livre la synthèse impossible à laquelle il choisit de se soumettre et qu'il appelle, de façon pompeuse, "l'imaginaire occidental »: il s'agit de tourner le dos, résolument, à l'avenir. Dans les dernières décennies surtout, l'avenir n'a servi qu'à alimenter des rêves qui ont fait crûment déchanter, et il a abusé ainsi de ce dont l'imaginaire occidental est capable; ou plutôt, mais ce n'est guère mieux, l'avenir attendu a effectivement accompli le destin de cet imaginaire précisément en tant qu'occidental, c'est-à-dire brillant du côté du soleil couchant et distillant donc un parfum de fin de partie, en se donnant, "dans une sorte d'intuition géniale", avec le nom du « couchant » la mort comme horizon (p. 13). Une compréhension à la fois moins superficielle et plus critique de la signification même du mot "Occident » est présentée par Peter Sloterdijk dans Le palais de cristal. À l'intérieur du capitalisme planétaire (Paris, Maren Sell Éditeurs, 2006; original allemand 2005). Ce livre reprend, sous une forme modifiée, la partie conclusive de Sphères II, Globes, Macrosphérologie, un livre publié en 1999 et dont la traduction française prochaine est annoncée. Le vaste projet des Sphères - Sphères I, Bulles, Microsphérologie (1998, traduction française 2002), Globes, et Sphères III, Écumes, Sphérologie plurielle (2004, traduction française 2005), trois volumes qui totalisent plus de 2500 pages - est une tentative de configurer le narratif et le philosophique I'un avec I'autre pour faire en sorte qu'il s'agisse de plus et d'autre chose que de la simple addition de deux visions de borgne, et de fournir, avec les moyens d'un grand récit d'inspiration philosophique, les contours d'une théorie du temps présent d'une manière autrement plus fouillée et complète que ce qui est offert dans Raconter et mourir. La façon dont Sloterdijk réclame et défend la nécessité des "grands récits" encore aujourd'hui est autrement plus convaincante que les accents éplorés de Hentsch sur leur absence actuelle et l'injonction de se défaire des emprises d'un futur qui tourne si vite à l'obsession. Mais ce livre pour bourlingueur fatigué ne fait que proposer un changement d'obsession: il entend procurer un bain d'étrangeté à même le familier devenu insipide à force d'être évident.

Les rubriques suivantes servent de titres aux cinq parties du livre: l. L'immortalité et la vie (p. 35-90); 2. L'épreuve de la connaissance (p. 91-196); 3. La vérité ou la mort (p. 197-259); 4. Héroïsme et vérité (p. 261-326); 5. L'irruption du doute (p. 327-415). Il est question d'Ulysse, d'Énée, et de Gilgamesh (partie I); du récit biblique de la Genèse, d'Hésiode, d'CEdipe-roi, d'Antigone et du Banquet de Platon (partie 2); de I'Évangile et des Confessions d'Augustin (partie 3); de la Chanson de Roland, de Perceval, de Lancelot, de Tristan et de la Divine Comédie de Dante (partie 4); de Rabelais, du Don Quichotte de Cervantès, de Hamlet et de Descartes (partie 5).

Si l'auteur achoppe sur le paradoxe qu'il nomme "récit de la vérité » qui ne vit que du dualisme oppositionnel temps (= récit)/éternité (= vérité), allant même jusqu'à en faire l'énigme centrale, selon lui à peine avouable, du christianisme, c'est qu'il oublie que « récit et vérité » n'est pas l'équivalent de " raconter et mourir ». Pareil rapprochement signale - et rappelle aussi - que la narration est travaillée de I'intérieur par ce qui la dépasse, par quelque chose d'inassimilable qui lui confère pourtant un ordonnancement qui vaut comme sens.

La préférence de l'auteur pour la thématique fait qu'il néglige l'importance de l'écriture, même s'il en parle souvent. Mais ces nombreux développements tournent facilement à la surenchère et ne parviennent pas à faire ombrage à la thématique du "et» dans le titre choisi: Raconter et mourir. Si cet «et" n'est d'aucun temps, ce n'est pas par l'effet d'un coefficient d'éternité qui tournerait le dos à I'histoire, mais c'est bien plutôt qu'il est de tous les temps et qu'il se retrouve donc, du moins dans l'imaginaire occidental qui est I'unique souci de l'auteur, dans les récits qu'il choisit d'analyser et qui montrent à quel point, dans cet ailleurs à l'occidentale, la source ne bruit que dans les marges. Seraitce donc que la mise en récit d'une vérité fait d'emblée de celle-ci une chimère et signale l'échec toujours annoncé d'une identité qui tenterait en vain de s'arracher à l'éphémère de la vie?

L'auteur tente d'endiguer son ambitieux projet - rien moins qu'un retour aux sources de l'imaginaire occidental - en s'arrêtant à Descartes chez qui sa lecture " trouve matière ou prétexte à conclure » (p. 397) ce pèlerinage aux sources. Tout ce qui vient après Descartes ne peut être que "faille mortelle dans la vérité » que Descartes entendait pourtant "sauver» (p. 415). Après Descartes, il n'y aurait place que pour des "ambitions démesurées et des espoirs destructeurs "; il est donc plus que temps, selon l'auteur, de tirer enfin les conclusions qui s'imposent quant à la croyance au progrès qui "s'étiole" sous nos yeux aujourd'hui, et de mieux discerner «ce qu'il en coûte d'avoir cru pouvoir rapatrier en ce monde la promesse de salut et la vérité que le christianisme réservait pour l'au-delà de la mort» (p. 415). C'est sur ce vœu que se clôt cette "série d'essais sur l'imaginaire occidental » (p. 4) qui témoigne une fois de plus - une fois de trop! - que nous vivons bien dans une culture qui ne peut pratiquement pas parler du plus manifeste si ce n'est sous la forme de la distinction grossière entre la bonne et la mauvaise ambiance.

La question qui demeure tout au long de la lecture de ce livre est celle-ci: I'auteur a-t-il vraiment quelque chose de neuf à dire, ou s'est-il plutôt contenté de citer une multitude d'auteurs dans le but d'égayer quelque peu un propos franchement répétitif qui se complaît et s'épuise à dénoncer un imaginaire « dominé par la peur du manque, la hantise de la maladie et le refus de la mort " (p. 421)? Ce livre est en tout cas une forte incitation à changer sa liste de lectures.

Raconter et mourir - c'est-à-dire, selon le "post-scriptum» du livre achevé d'imprimer en septembre 2002, "mourir apaisé d'avoir vécu et transmis» (p. 420). C'est, malgré tout, ce qu'il y a encore de mieux à espérer pour l'Auteur qui devait décéder moins de trois ans plus tard, le 7 juillet 2005.

Maurice Boutin

\section{BAUD, René-Claude \\ Ce qui remonte de l'ombre} Itinéraire d'un soignant

Paris, Bayard, 2006, 163 p.

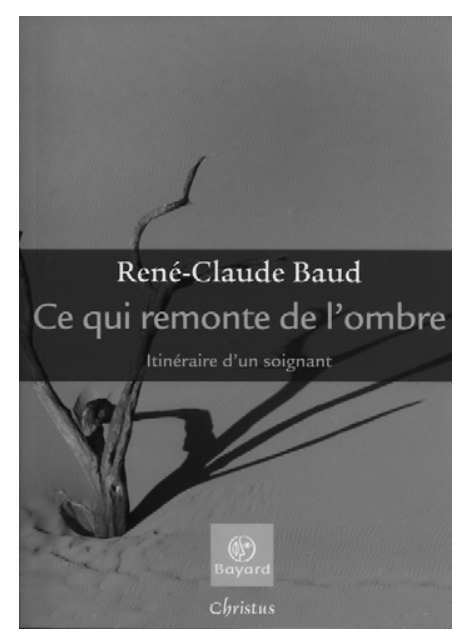

Jésuite (p. 11), diplômé en philosophie et en théologie (p. 63), ancien aumônier dans un collège pour adolescents (p. 62 et 153), aide-soignant de nuit, pendant vingt ans (p. 46), auprès de malades atteints du sida (p. 10), responsable de la formation d'accompagnants en soins palliatifs ( $p .126$ ) et fondateur de l'association Albatros à Lyon (quatrième de couverture), 
René-Claude Baud se livre dans ce texte à une relecture de vie, présentée sous forme de réflexions. Bien qu'il puisse se référer à une riche expérience, l'auteur ne se présente jamais comme un maître à penser ou un modèle. Les modèles, nous ditil, sont inutiles (p. 60). Chacun doit suivre son propre chemin. Quant au maître, s'il y en a un, c'est le malade et il est d'autant plus exigeant et efficace qu'il ne le sait pas (p. 30). Selon l'auteur, ce sont en effet les malades qui lui ont appris d'autres langages que celui des mots, d'autres silences que le vide des mots; ce sont eux qui lui ont appris à se risquer dans les langages du corps, du regard, du toucher et du corps à corps (p. 33). Grâce aux malades, il a appris la langue de la proximité, celle des yeux, des mains, de la caresse légère, cette langue que chacun comprend dans sa propre langue, qu'il soit sourd ou même confus (p. 63). II a appris qu'un sourire et un geste de la main en disent tellement plus que les mots usés du quotidien (p. 76). Ce langage de l'apprentissage traverse tout le livre. Voici, par exemple, comment il décrit son arrivée à l'hôpital comme aide-soignant: "Dans cette situation nouvelle, l'acquis antérieur ne m'était plus d'aucune ressource: les comportements anciens, pourtant éprouvés comme efficaces, se révélaient caducs. Tel l'immigré, j'étais dans la nécessité d'apprendre une nouvelle langue » (p. 27-28). Et cette langue, il l'a apprise. II a appris les formes d'une présence impuissante et silencieuse (p. 47). II a appris à être plus modeste. II a accepté de ne rien savoir d'un être humain, de sa souffrance, de son chemin. II a appris aussi à découvrir de nouvelles expressions d'humanité et de tendresse, comme l'amitié entre homosexuels ou entre gens marginaux et psychologiquement fragiles (p. 43). Il a appris une autre manière d'aider: non plus " faire pour ", mais "être avec", inventer une autre manière de vivre ensemble (p. 65). La fréquentation des malades lui a aussi progressivement dévoilé que le soignant n'accompagne pas qui il veut. En dépit des apparences, ce sont en effet les personnes soignées qui prennent l'initiative de l'accompagnement (p. 44), ce sont elles qui ont l'initiative des démarches importantes (p. 133). Reconnaître ce fait est essentiel, même si c'est un dur coup pour le narcissisme primaire de l'aide-soignant, car il y a toujours un danger d'encombrer le malade de son propre désir d'aider; il y a toujours un risque de se prendre pour un sauveur et de se croire indispensable sur le chemin d'un autre (p. 49-50).
Les aides-soignants à l'affût de recettes et de techniques faciles ne trouveront pas ce qu'ils cherchent dans ce livre, car l'auteur ne cesse de convier chacun à faire son propre apprentissage, à poursuivre son propre chemin. Quant aux intellectuels préoccupés par la seule rigueur des raisonnements éthiques, ils seront sans doute déçus ou surpris par l'argumentation sommaire qui justifie le refus de l'euthanasie (p. 140-147). Enfin, ceux qui ne sont pas chrétiens liront peut-être avec froideur les modestes réflexions théologiques qui accompagnent cette relecture de vie. Toutefois, tous partageront sûrement son refus actif et pacifique de voir mourir des gens dans la souffrance physique et psychologique, et ce dans l'indifférence du reste de la société. En définitive, tous ceux qui s'interrogent sur l'accompagnement des malades en fin de vie et qui veulent savoir comment il est possible de parler de ce qui remonte de l'ombre, sans pour autant prétendre connaître la lumière de l'aube, trouveront dans ce livre de quoi éclairer, comme une veilleuse dans la nuit, leurs propres questionnements et leurs propres cheminements.

\section{Jean-Jacques Lavoie}

DANEAULT, Serge, avec la collaboration de Véronique LUSSIER et de Suzanne MONGEAU

\section{Souffrance et médecine}

Québec, Presses de l'Université du Québec, collection «Santé et Société », 2006, 159 p.

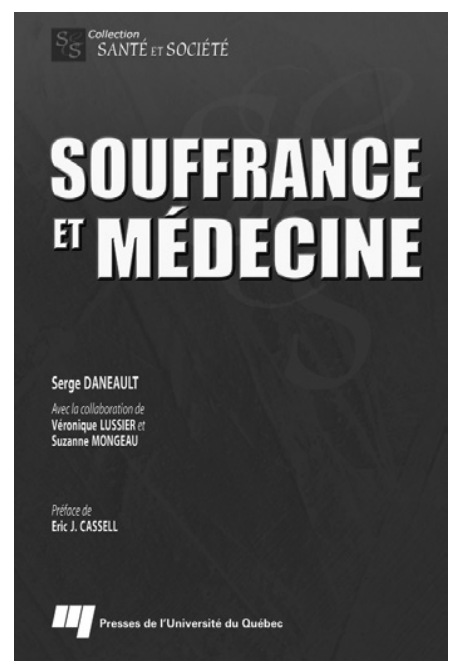

Préfacé par Eric J. Cassell, un des premiers médecins américains à avoir produit des travaux sur le phénomène de la souffrance, le livre Souffrance et médecine est un ouvrage captivant, mais aussi troublant par moments. On en termine la lecture avec un goût un peu amer en bouche face aux constats posés par l'équipe de recherche dirigée par Serge Daneault, médecin et professeur à la Faculté de médecine de l'Université de Montréal. Ce livre s'intéresse de près au phénomène de la souffrance dans les services de santé, et plus particulièrement à la souffrance des personnes touchées par une maladie terminale et à celle des soignants. Il est divisé en neuf chapitres qui, comme le mentionne d'ailleurs l'auteur principal, peuvent être lus indépendamment I'un de l'autre sans que cela nuise à la compréhension du texte. Le premier chapitre propose une description de la problématique et de la méthodologie de la recherche dont les résultats sont rapportés dans ce volume. Bien que s'adressant en premier lieu aux chercheurs qui prendront connaissance de cet ouvrage, ce chapitre apparaît néanmoins suffisamment accessible, dans l'ensemble, pour des lecteurs qui ne sont pas familiers avec l'univers de la recherche. Il campe très bien le contexte et l'envergure de cette recherche menée principalement auprès de vingt-six personnes gravement malades provenant de trois hôpitaux montréalais et de cinquante-trois soignants recrutés dans un milieu universitaire de la métropole. Les chapitres 2 à 4 s'intéressent à l'expérience de la souffrance du point de vue des personnes gravement malades en proposant des réponses aux questions qui suivent: "Quelles sont les sources de la souffrance chez les grands malades?", "Qu'est-ce que souffrir?" et "Comment la souffrance est-elle prise en charge par les services de santé ? "Devant les jugements souvent très sévères qui sont posés envers les soignants et le système de santé dans ces trois chapitres, il devenait nécessaire de recueillir le point de vue des soignants sur la souffrance des personnes malades ainsi qu'au sujet des obstacles au soulagement de la souffrance dans le système de santé, ces thèmes faisant respectivement l'objet des chapitres 5 et 6 . Les constats provenant de ces deux chapitres conduisent à une analyse fine et nuancée de la souffrance des soignants eux-mêmes, proposée au septième chapitre. Finalement, un huitième chapitre traite de la place des soins palliatifs au sein de nos services de santé et de notre société et un neuvième chapitre fait office de conclusion en offrant notamment quelques pistes de solutions proposées par les soignants interrogés.

Une des caractéristiques contribuant à la qualité de cet ouvrage est le recours abondant aux témoignages directs des personnes touchées par la maladie terminale et des soignants. Contrairement à ce que l'on voit trop souvent dans des rapports de recherche qualitative où très peu d'espace est accordé aux propos des gens interrogés, l'auteur et ses collaboratrices n'ont pas hésité à insérer de nombreux extraits des témoignages recueillis. C'est donc à travers ces témoignages que Souffrance et médecine pose un regard lucide, et souvent dérangeant, sur le système de santé québécois, et que le lecteur est amené à réfléchir à des questions dont les enjeux sont considérables. Par exemple, comment un système de santé dont on s'attend notamment à ce qu'il apaise la souffrance des personnes malades à défaut de la neutraliser totalement, en est-il arrivé à devenir une des causes de souffrance pour ces personnes? Ou encore, comment humaniser les soins alors même que les soignants affirment que les hôpitaux sont gérés comme des "fabriques de petits pois ", métaphore lourde de sens que les auteurs reprennent à différentes reprises dans le livre? Les quelques pages qui concernent de manière directe les soins palliatifs et qui soulignent à la fois leur raison d'être et leur grande fragilité dans le système de santé actuel, suscitent elles aussi un bon nombre de questions et de réflexions chez le lecteur.

Pour les gens qui fréquentent assidûment le système de santé en tant qu'usagers ou employés, les constats offerts par Souffrance et médecine n'apparaîtront pas aussi surprenants et bouleversants qu'à un observateur extérieur. Toutefois, par l'analyse systématique et rigoureuse qu'il propose de la souffrance des uns et des autres, tout comme par le regard critique qu'il porte sur nos services de santé, ce livre possède le grand mérite de légitimer des impressions et des sentiments qui en habitent plusieurs. Au-delà de cette légitimation qui pourrait demeurer stérile si elle n'était accompagnée de propositions de changements, ce livre offre des pistes d'intervention pour les soignants et, comme il a été mentionné précédemment, sa conclusion ouvre sur quelques pistes de solutions intéressantes et porteuses d'espoir pour un système qui en a bien besoin.

Un autre des grands mérites de ce livre est d'avoir réussi à demeu- 